\title{
Uma Investigação sobre Recomendações de Melhoria Baseadas em Aspectos Culturais para Apoiar Iniciativas de Melhoria de Processo de Software
}

\author{
Odette M. Passos, Arilo C. Dias-Neto, Raimundo da S. Barreto \\ Instituto de Computação - Universidade Federal do Amazonas (UFAM) \\ Av. Rodrigo Otávio, 3000 - Campus Universitário - 69077-000 - Coroado I \\ Manaus - AM - Brasil \\ \{odette, arilo, rbarreto\} aicomp.ufam.edu.br
}

\begin{abstract}
Resumo. Recomendações de melhoria (RMs) fornecem possíveis orientações que ajudam as organizações no alcance de seus objetivos e podem estar direcionadas a diferentes contextos. Neste trabalho, o foco é a cultura organizacional, que guia o funcionamento de uma organização e, ao mesmo tempo, influencia a forma como os profissionais reagem a iniciativas de melhoria de processo de software. Este artigo apresenta os resultados de uma revisão sistemática que identificou 100 RMs para que organizações de software obtenham sucesso na implementação dessas iniciativas. Estas RMs foram organizadas em três grupos, representando fatores humano, social e organizacional. As análises realizadas apresentam as RMs mais citadas na literatura técnica em uma perspectiva individual e por grupos.
\end{abstract}

\begin{abstract}
Recommendations for improvement (RIs) provide useful guidelines that help organizations in achieving their goals and can be assigned to different contexts. In this paper, the focus is on organizational culture that guides the functioning of an organization and, at the same time, influences how professionals react to software process improvement initiatives. This paper presents the results of a systematic review which identified 100 RIs to support software organizations to succeed in implementing these initiatives. These RIs were organized in three groups, representing human, social, and organizational factors. Analysis presents the most cited RIs in the technical literature in two perspectives: individual and team.
\end{abstract}

\section{Introdução}

Com a crescente demanda por qualidade dos produtos de software, a adoção de modelos de maturidade, normas de qualidade e guias de boas práticas na definição de processos têm se tornado cada vez mais frequentes. Esses modelos, normas e guias (comumente chamados de iniciativas de melhoria de processo de software - MPS) procuram melhorar o desempenho das organizações com respeito a custo, prazo, produtividade, satisfação do cliente e retorno do investimento e, consequentemente, aumentar sua vantagem competitiva [Santos, 2011].

Diversos modelos de maturidade, como CMMI-DEV [SEI, 2006] e MPS.BR [SOFTEX, 2009], e normas, como ISO/IEC 15504 [ISO/IEC, 2003] e ISO/IEC 12207 [ISO/IEC, 2008], de apoio à qualidade de software, têm sido aplicados frequentemente em organizações de software com o propósito de organizar o processo de trabalho e 
obter melhores resultados para o desenvolvimento de software. Contudo, a quantidade de organizações que adotam esses modelos/normas ainda é uma parcela reduzida da população total de organizações de software. Um dos motivos pode ser as dificuldades encontradas durante a implementação da iniciativa de MPS [Montoni e Rocha, 2011].

Cada organização possui políticas e crenças específicas, que caracterizam a sua cultura organizacional, e devem ser levadas em consideração para que as melhorias sejam bem aceitas e realmente contribuam com um desenvolvimento mais eficiente [Rodrigues e Kirner, 2010]. A cultura organizacional (CO) é um fator que pode influenciar a forma como os profissionais reagem a iniciativas de MPS [Dyba, 2002]. A organização precisa utilizar os insights da $\mathrm{CO}$ para melhor implementar essas iniciativas, isto é, a alta direção precisa assegurar que as práticas de MPS estejam de acordo com a CO estabelecida na organização [Muller et al., 2009].

Schein (2009) define CO como um conjunto de características adotadas pela organização que dizem respeito a padrões de comportamento, ideologias, memórias coletivas, normas e costumes, estilo de gerência e políticas administrativas. Essas características são seguidas por todos os membros da organização para atingir seu objetivo. Cameron e Quinn (2006) definem CO da seguinte forma: "como as coisas são feitas por aqui”".

Organizações costumam se basear em recomendações de melhoria (RMs) disponíveis, geralmente, na literatura técnica, para apoiar a implementação de iniciativas de MPS. As RMs fornecem orientações relacionadas a aspectos considerados relevantes para uma organização e podem ser baseadas em conhecimento adquirido com experiências anteriores [Barreto e Rocha, 2012]. A implantação dessas RMs podem reduzir problemas e riscos, induzir a uma gestão mais eficiente e auxiliar as organizações a alcançarem seus objetivos e metas. No entanto, estas RMs podem apresentar direcionamentos sob diferentes perspectivas, sejam organizacionais, técnicas, culturais, humanas, ferramentais, dentre outras possibilidades. Neste trabalho, o escopo está direcionado à análise da perspectiva da CO sobre as iniciativas de MPS. A partir desse resultado, foi possível relacionar RMs que causam um impacto direto no sucesso da iniciativa de MPS e podem ser aplicadas a organização com diferentes características.

Nesse contexto, este trabalho apresenta um total de 100 RMs para auxiliar organizações de software a obterem sucesso na implementação de uma iniciativa de MPS. As RMs foram identificadas por meio da execução de um estudo secundário baseado em revisão sistemática da literatura [Kitchenham, 2004] e foram organizadas em três grupos representando fatores humano, social e organizacional. Uma análise foi realizada, com os dados obtidos, baseada nas RMs mais citadas pelos estudos identificados, em uma perspectiva individual e por grupos.

Há diversos trabalhos relacionados à RMs, porém com propósitos bastante variados. O trabalho de Barcellos et al. (2010), por exemplo, indica um conjunto de RMs para medição de software adequada ao controle estatístico de processos. Em [Souza et al., 2009] é apresentado um conjunto de recomendações para avaliação conjunta dos modelos de maturidade CMMI e MPS.BR, realizada em uma empresa brasileira. Outros trabalhos citam RMs para atingir o Nível A [Souza et al., 2010] ou Nível D [Ricardo e Correa, 2011] do modelo MPS.BR. Embora existam diversos trabalhos que sugerem RMs, não foram identificados trabalhos anteriores que 
apresentam uma abordagem para apoiar a implementação de uma iniciativa de MPS, a partir da $\mathrm{CO}$, por meio de um conjunto de RMs consolidadas.

Este artigo está organizado da seguinte forma: além desta introdução, ele é formado por outras quatro seções. A Seção 2 descreve a metodologia utilizada para a condução da revisão sistemática. A Seção 3 apresenta as recomendações de melhoria identificadas a partir da revisão sistemática. A Seção 4 apresenta uma análise nos dados coletados. Por fim, as considerações finais são discutidas na Seção 5.

\section{Revisão Sistemática para a Identificação de Recomendações de Melhoria}

Revisão sistemática da literatura (RSL) é um meio de identificar, avaliar e interpretar toda pesquisa disponível e relevante sobre uma questão de pesquisa, um tópico ou um fenômeno de interesse, e têm por objetivo apresentar uma avaliação justa de um tópico de pesquisa, usando uma metodologia confiável, rigorosa e auditável [Kitchenham, 2004].

A aplicação de RSL requer que seja seguido um conjunto sequencial e bem definido de passos, segundo um protocolo de pesquisa desenvolvido apropriadamente. Este protocolo é construído considerando um tema específico que representa o elemento central da investigação. Os passos da pesquisa, as estratégias definidas para coletar as evidências e o foco das questões de pesquisa são definidos explicitamente, de tal forma que outros pesquisadores sejam capazes de reproduzir o mesmo protocolo de pesquisa e, também, de julgar a adequação dos padrões adotados no estudo [Biolchini et al., 2005].

O processo para a condução da RSL realizada neste trabalho envolveu três etapas: Planejamento, Execução e Publicação dos Resultados [Kitchenham, 2004]. Detalhes sobre esta RSL encontram-se em [Passos et al., 2012].

\subsection{Planejamento da Revisão Sistemática}

Nesta etapa foi definido o protocolo de pesquisa, que consiste em definir o objetivo do estudo, especificar a questão principal e as questões secundárias da pesquisa, formular as strings de busca, além de mencionar os procedimentos de extração dos dados e os critérios de inclusão e exclusão de cada publicação. A estrutura do protocolo foi baseada em [Kitchenham, 2004], conforme descrito a seguir:

- Objetivo: Analisar relatos de experiências e publicações científicas através de um estudo baseado em revisão sistemática com o propósito de identificar recomendações de melhoria baseadas na cultura organizacional em relação à contribuição para o sucesso da implementação de uma iniciativa de MPS do ponto de vista dos pesquisadores no contexto acadêmico e industrial.

- Questões: pretende-se obter respostas para as questões a seguir:

○ Questão Principal: Quais as RMs que têm relação com a CO em uma iniciativa de MPS?

* Questão Secundária 1: Quais das RMs identificadas que promoveram o sucesso da adoção/implementação da iniciativa de MPS?

* Questão Secundária 2: Existe evidência de que as RM foram avaliadas?

* Questão Secundária 3: Como as RMs estão distribuídas em relação ao número de citações entre os estudos selecionados?

- Escopo da Pesquisa: Para delinear o escopo da pesquisa, foram estabelecidos critérios para garantir de forma equilibrada a viabilidade da execução, acessibilidade 
aos dados e abrangência do estudo. A pesquisa dar-se-á a partir de bibliotecas digitais através das suas respectivas máquinas de busca e, quando os dados não estiverem disponíveis eletronicamente, através de consultas manuais.

- Critérios Adotados para Seleção das Fontes: Foram selecionadas para consulta bibliotecas digitais que permitam o uso de expressões lógicas ou mecanismo equivalente em suas máquinas de busca. Além disso, foram consideradas conferências patrocinadas pela Sociedade Brasileira de Computação (SBC) na área de Engenharia de Software. Somente trabalhos escritos em português e inglês foram selecionados.

- Métodos de Busca das Publicações: As fontes digitais foram acessadas via Web. Caso não fosse possível obter o artigo completo através dos sites de busca, os autores dos artigos foram contatados por e-mail. As fontes digitais consultadas foram: IEEE Computer Science Digital Library (IEEExplorer) e Scopus (que segundo a Editora Elsevier (2013), cobre outras bibliotecas, como ACM Digital Library, Springer Link e ScienceDirect/Elsevier). Além disso, foram consultadas manualmente as publicações das seguintes conferências: Simpósio Brasileiro de Engenharia de Software (SBES), Simpósio Brasileiro de Qualidade de Software (SBQS) e Workshop Anual do MPS (WAMPS). As strings de busca foram definidas de acordo com dois dos quatro aspectos indicados em [Peterson et al., 2008] para formar strings de busca: População e Intervenção, conforme a Tabela 1.

Tabela 1. Expressão de busca utilizada para encontrar as publicações

População: iniciativas de melhoria de processo de software (e sinônimos):

- Palavras-Chave: "SPI" OR "process improvement" OR "process enhancement" OR "process change" OR "process assessment" AND "software".

Intervenção: publicações que fazem referências à cultura organizacional (e sinônimos):

- Palavras-Chave: "organizational culture" OR "organisational culture" OR "organizational change" OR "organisational change" OR "organizational behavior" OR "organisational behavior" OR "organizational behaviour" OR "organisational behaviour" OR "organizational issues" OR "organisational issues" OR "organizational politics" OR "organisational politics" OR "organizational factors" OR "organisational factors" OR "organizational type" OR "organisational type" OR "subculture" OR "cultural change" OR "culture change" OR "cultural management" OR "culture management" OR "change management" OR "culture profile" OR "cultural factors" OR "cultural influences".

- Procedimentos de Seleção e Critérios: A estratégia de busca foi aplicada para identificar as publicações em potencial. Elas foram selecionadas pelos pesquisadores por meio da verificação dos critérios de inclusão/exclusão estabelecidos. Para diminuir o risco que uma publicação fosse excluída prematuramente em uma das etapas do estudo, sempre que existia dúvida sobre a inclusão, a publicação não foi excluída. A seleção dos estudos foi feita em 4 etapas:

I. Seleção e catalogação preliminar dos dados: A seleção preliminar das publicações foi feita a partir da aplicação da expressão de busca às fontes selecionadas. Cada publicação foi catalogada em um repositório para análise posterior;

II. Seleção dos dados relevantes - [ $\mathbf{1}^{\mathbf{0}}$ filtro]: A seleção preliminar não garante que todo o material coletado seja útil no contexto da pesquisa. Dessa forma, após a identificação das publicações através dos mecanismos de buscas, foi lido o título, o resumo e as palavras-chave e analisados seguindo os critérios de inclusão e exclusão do $1^{\text {o }}$ filtro. Basicamente, para que uma publicação fosse incluída neste filtro, era necessário que o contexto das palavras levasse a crer que a publicação 
cita uma iniciativa de MPS e faz referências à CO. Caso um estudo não atendesse a esse critério, este era consequentemente excluído do passo seguinte da análise.

III. Seleção dos dados relevantes - [ $2^{\circ}$ filtro]: Apesar de limitar o universo de busca, o $1^{\circ}$ filtro empregado não garante que todo o material coletado seja útil no contexto da pesquisa. Por isso, após a leitura na íntegra dos artigos selecionados no $1^{\circ}$ filtro, foi verificado se de fato as publicações identificadas contextualizavam uma iniciativa de MPS com foco em CO. Caso um estudo não atendesse a esse critério, este era consequentemente excluído do passo seguinte da análise.

IV. Seleção dos dados relevantes - [ $3^{\circ}$ filtro]: $O$ objetivo foi identificar quais estudos mencionavam RMs que estavam relacionadas com iniciativas de MPS e CO.

- Procedimentos para Extração dos Dados: Os dados extraídos das publicações selecionadas foram armazenados em um repositório e contêm as informações apresentadas na Tabela 2 (uma instância desta Tabela encontra-se em [Passos et al., 2012].

Tabela 2. Campos de coleta de dados

\begin{tabular}{|c|c|}
\hline Título: & indica o título do artigo. \\
\hline Referência completa: & informações completas sobre os autores, ano e local de publicação. \\
\hline Palavras-chave: & lista as palavras-chaves. \\
\hline $\begin{array}{l}\text { Impacto da CO } x \text { MPS } \\
\text { (positivo } x \text { negativo): }\end{array}$ & $\begin{array}{l}\text { indica se a relação entre CO e iniciativas de MPS foi positiva ou negativa, isto é, } \\
\text { se a cultura influencia ou não na adoção de uma iniciativa de MPS. }\end{array}$ \\
\hline Recomendações de melhoria: & sugestões propostas para a melhoria da CO em iniciativas de MPS. \\
\hline $\begin{array}{l}\text { Resultado das } \\
\text { recomendações (sim ou não): }\end{array}$ & $\begin{array}{l}\text { indica se as recomendações de melhoria promoveram ou não o sucesso na } \\
\text { adoção/implementação da iniciativa de MPS. }\end{array}$ \\
\hline $\begin{array}{l}\text { Validação das } \\
\text { recomendações (sim ou não): }\end{array}$ & $\begin{array}{l}\text { se sim, qual tipo de estudo foi utilizado: estudo de caso, experimento, pesquisa de } \\
\text { opinião, dentre outros. }\end{array}$ \\
\hline $\begin{array}{l}\text { Modelo da cultura } \\
\text { organizacional: }\end{array}$ & $\begin{array}{l}\text { nome do autor do modelo para análise da } \mathrm{CO} \text { que foi utilizado no artigo, se } \\
\text { houver algum. }\end{array}$ \\
\hline $\begin{array}{l}\text { Iniciativa de melhoria de } \\
\text { processo de software: }\end{array}$ & $\begin{array}{l}\text { descrição das características da iniciativa de melhoria de processo de software } \\
\text { descrita no artigo, como: modelo de maturidade, normas ou técnicas. Informar se } \\
\text { a iniciativa de MPS foi descrita em uma organização individual ou num grupo de } \\
\text { organizações. }\end{array}$ \\
\hline Organização: & $\begin{array}{l}\text { descrição das características das organizações onde foram realizados os } \\
\text { experimentos, como: localização, tamanho (se pequena, média, grande ou se } \\
\text { corporação) e nível de maturidade. }\end{array}$ \\
\hline Citação de outros trabalhos: & $\begin{array}{l}\text { descrição dos artigos que foram citados na publicação e que estejam relacionados } \\
\text { com CO e MPS. }\end{array}$ \\
\hline Perspectivas futuras: & questão de pesquisa sugerida para trabalhos futuros, se houver alguma. \\
\hline
\end{tabular}

\subsection{Condução da Revisão Sistemática e Publicação dos Resultados}

Após a etapa de planejamento, a string de busca foi executada nas fontes definidas no período de maio a junho de 2012. As publicações foram selecionadas de acordo com os critérios de inclusão/exclusão estabelecidos no protocolo.

No total, 245 publicações foram identificadas, das quais 34 foram inicialmente excluídas, pois estavam duplicadas. Das 211 publicações restantes, todas foram analisadas e 26 discorrem sobre CO e MPS. Dessas, 11 citam RMs, como pode ser observado na Figura 1. Das 680 publicações pesquisadas manualmente, 7 passaram pela expressão de busca (porém nenhuma publicação passou pelo $3^{\circ}$ filtro). A lista completa dos artigos identificados e as informações registradas durante a realização da RS podem ser encontradas em [Passos et al., 2012]. 


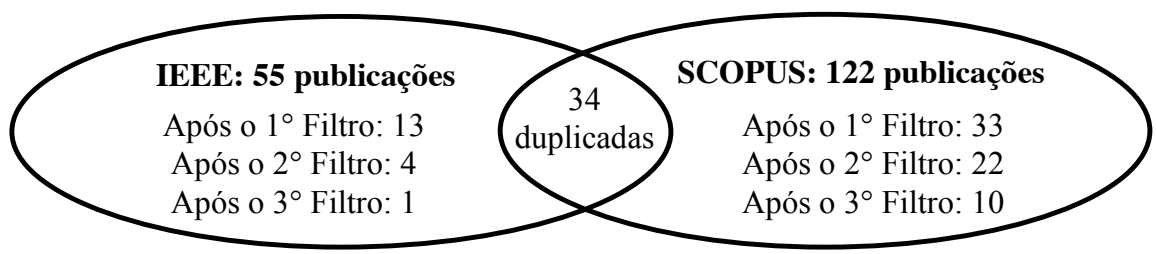

Figura 1. Distribuição dos artigos nas máquinas de busca

Das 11 publicações identificadas (Tabela 3), foi possível extrair 100 RMs (veja Seção 3) que estão relacionadas com a CO em uma iniciativa de MPS (questão principal de pesquisa). Essas RMs precisam estar alinhadas à realidade da empresa considerando suas características, necessidades e restrições.

Tabela 3. Publicações selecionadas após o $3^{\circ}$ filtro

\begin{tabular}{|c|c|c|c|}
\hline $\mathbf{N}^{\circ}$ & Título & Autores & Ano \\
\hline 1 & $\begin{array}{l}\text { Software Process Improvement: A Organizational Change that } \\
\text { Need to be Managed and Motivated }\end{array}$ & Ferreira, M.; Wazlawick, R. & 2011 \\
\hline 2 & $\begin{array}{l}\text { Software Process Improvement as Organizational Change: A } \\
\text { Metaphorical Analysis of the Literature }\end{array}$ & $\begin{array}{l}\text { Muller, S.; Mathiassen, L.; } \\
\text { Balshoj, H. }\end{array}$ & 2010 \\
\hline 3 & $\begin{array}{l}\text { Exploring the Relationship Between Organizational Culture and } \\
\text { Software Process Improvement Deployment }\end{array}$ & Shih, C-C.; Huang, S-J. & 2010 \\
\hline 4 & $\begin{array}{l}\text { Managing Cultural Variation in Software Process Improvement: } \\
\text { A Comparison of Methods for Subculture Assessment }\end{array}$ & $\begin{array}{l}\text { Muller, S.; Kraemmergaard, P.; } \\
\text { Mathiassen, L. }\end{array}$ & 2009 \\
\hline 5 & $\begin{array}{l}\text { Problems in Measuring Effectiveness in Software Process } \\
\text { Improvement: A Longitudinal Study of Organizational Change at } \\
\text { Danske Data }\end{array}$ & Iversen, J.; Ngwenyama, O. & 2006 \\
\hline 6 & $\begin{array}{l}\text { An Empirical Investigation of the Key Factors for Success in } \\
\text { Software Process Improvement }\end{array}$ & Dyba, T. & 2005 \\
\hline 7 & Managing Change in Software Process Improvement & $\begin{array}{c}\text { Mathiassen, L.; } \\
\text { Ngwenyama, O.; Aaen, I. }\end{array}$ & 2005 \\
\hline 8 & Methodological Issues in a CMM Level 4 Implementation & $\begin{array}{l}\text { Antoniol, G.; Gradara, S.; } \\
\text { Venturi, G. }\end{array}$ & 2004 \\
\hline 9 & $\begin{array}{l}\text { Competing Values in Software Process Improvement: An } \\
\text { Assumption Analysis of CMM From an Organizational Culture } \\
\text { Perspective }\end{array}$ & $\begin{array}{l}\text { Ngwenyama, O.; } \\
\text { Nielsen, P. }\end{array}$ & 2003 \\
\hline 10 & The Role of Culture in Successful Software Process Improvement & $\begin{array}{l}\text { Sharp, H.; Woodman, M.; } \\
\text { Hovenden, F.; Robinson, H. }\end{array}$ & 1999 \\
\hline 11 & Which Comes First, the Organization or Its Processes? & Curtis, B. & 1998 \\
\hline
\end{tabular}

\section{Recomendações de Melhoria relacionadas à CO para Iniciativas de MPS}

Recomendações de melhoria são sugestões que ajudam as organizações na tomada de decisões e normalmente são baseadas em experiências anteriores [Barreto e Rocha, 2012]. Elas têm como objetivo fornecer possíveis orientações relacionadas a aspectos considerados relevantes para uma organização, potencializando os resultados esperados e, até mesmo, garantindo sua permanência no mercado. Assim, este trabalho identificou $100 \mathrm{RMs}$, baseadas na perspectiva da CO, para auxiliar as organizações de software na implementação de uma iniciativa de MPS.

Existem fatores que contribuem para o sucesso ou fracasso da implementação de uma iniciativa de MPS. Dentre eles, podem ser citados fatores humanos, sociais [Zoucas et al., 2012] e organizacionais [Dyba, 2005], pois uma iniciativa de MPS envolve pessoas com diferentes graus de conhecimento e objetivos. Neste contexto, as RMs identificadas neste trabalho foram organizadas em grupos que representam esses fatores (humano, social e organizacional) no processo de implementação da iniciativa de MPS. Para facilitar a organização das RMs identificadas, cada grupo (fator) está dividido em categorias que foram determinadas conforme a palavra-chave retirada do texto da 
própria RM. Assim, o fator humano $(\mathrm{FH})$ foi subdividido em 3 categorias, o fator social (FS) em 5 categorias e o fator organizacional (FO), em 5 categorias.

A Tabela 4 apresenta as RMs relacionadas ao grupo fator humano. Essas RMs podem ser definidas como novas qualificações e habilidades, tanto individuais quanto coletivas, e podem variar para atender desde as características da estrutura das organizações até características mais específicas da personalidade [Senge, 1998]. Na coluna "Citações" é mostrada a quantidade de publicações (dentre as 11) onde as RMs foram identificadas (em [Passos et al., 2012] encontra-se as publicações de onde as RMs foram retiradas).

Tabela 4. RMs do fator humano extraídas das publicações

\begin{tabular}{|c|c|c|}
\hline RMs & Descrição & Citações \\
\hline \multicolumn{3}{|c|}{ Equipes de Trabalho: } \\
\hline RM1 & $\begin{array}{l}\text { Definir uma equipe de garantia da qualidade, para apoiar a implementação da iniciativa de MPS. } \\
\text { A equipe deve ser composta por pessoas que (i) possuem senso de coordenação, (ii) sejam } \\
\text { experientes e qualificadas, (iii) sejam reconhecidas por ter um bom envolvimento com a alta } \\
\text { direção e (iv) consigam integrar os funcionários nos objetivos da implementação da estratégia. } \\
\text { Além disso, que inspire confiança, respeito e autoridade nos funcionários. É necessário, que } \\
\text { tenham conhecimento em software que possam contribuir para a melhoria do processo. }\end{array}$ & 11 \\
\hline RM2 & $\begin{array}{l}\text { Definir as competências, funções e responsabilidades dos membros das equipes para realizar os } \\
\text { papéis na implementação da iniciativa de MPS. }\end{array}$ & 8 \\
\hline RM3 & Possibilitar e incentivar o compartilhamento de ideias, experiências e conhecimentos sobre MPS. & 4 \\
\hline RM4 & $\begin{array}{l}\text { Criar diversos grupos especiais (com diferentes funções) que tenham responsabilidades e com } \\
\text { conhecimentos especializados para compor a estrutura organizacional. Definir o escopo e as } \\
\text { responsabilidades dos grupos de acordo com a experiência das pessoas que o compõe. }\end{array}$ & 3 \\
\hline RM5 & $\begin{array}{l}\text { Enfatizar e estimular o trabalho em equipe e o comprometimento dos colaboradores através do } \\
\text { desenvolvimento de um sistema de valores que promova forte identidade corporativa. }\end{array}$ & 2 \\
\hline RM6 & $\begin{array}{l}\text { Formar equipes com uma composição etária misturada, isto é, composta por jovens e por } \\
\text { pessoas mais velhas. }\end{array}$ & 1 \\
\hline RM7 & Valorizar a criatividade dos funcionários e capacitar os mais competentes. & 1 \\
\hline RM8 & $\begin{array}{l}\text { Definir como as equipes devem realizar as atividades durante o desenvolvimento de um } \\
\text { software. Para isso, a organização deve coordenar e gerenciar as atividades. }\end{array}$ & 1 \\
\hline RM9 & Coletar e analisar dados de desempenho das equipes para avaliar a competência. & 1 \\
\hline RM10 & $\begin{array}{l}\text { Assegurar que os grupos de trabalho possuam habilidades para compartilhar as informações e } \\
\text { coordenar suas atividades de forma eficiente. }\end{array}$ & 1 \\
\hline \multicolumn{3}{|c|}{ Relacionamento: } \\
\hline RM11 & $\begin{array}{l}\text { Respeitar a experiência das equipes de trabalho e reconhecer a relevância do conhecimento } \\
\text { sobre MPS na resolução de problemas. Usar os conhecimentos e as experiências para decidir, } \\
\text { agir e assumir responsabilidades pela iniciativa de MPS. }\end{array}$ & 3 \\
\hline RM12 & $\begin{array}{l}\text { Ter um relacionamento caracterizado pelo respeito mútuo, pela educação, amizade, simpatia e } \\
\text { ajuda. }\end{array}$ & 2 \\
\hline RM13 & Escolher pessoas que tenham qualidades como paciência e experiência para trabalharem juntas. & 1 \\
\hline RM14 & $\begin{array}{l}\text { Criar possibilidades para que as equipes tenham uma relação (conexões) entre si, podendo ser } \\
\text { através de programas de rotatividade, promoção ou estratégias de recrutamento. }\end{array}$ & 1 \\
\hline RM15 & Conhecer e compreender os problemas enfrentados por todos os grupos de trabalho. & 1 \\
\hline RM16 & Dispor de tempo para atender às solicitações de dúvidas das equipes. & 1 \\
\hline RM17 & Dar ênfase nas tarefas e não no indivíduo que a executa. & 1 \\
\hline \multicolumn{3}{|c|}{ Características Pessoais: } \\
\hline RM18 & $\begin{array}{l}\text { Definir os líderes responsáveis pela implementação da iniciativa de MPS. Estes devem lidar } \\
\text { com o desenvolvimento e definição do estilo de gestão, do meio de comunicação e da interação } \\
\text { social dentro da organização. Além disso, devem ser pessoas com alta credibilidade dentro da } \\
\text { organização, com alto poder de liderança, organizados, ter sua competência reconhecida, ser } \\
\text { bons coordenadores e estarem disponíveis. }\end{array}$ & 5 \\
\hline RM19 & $\begin{array}{l}\text { Ter como características o comprometimento, a disponibilidade, a integridade e participar } \\
\text { ativamente na implementação da iniciativa de MPS. }\end{array}$ & 2 \\
\hline RM20 & Assumir compromissos e concordar com o que foi definido. & 1 \\
\hline RM21 & Verificar se o orgulho profissional faz parte da personalidade dos membros das equipes. & 1 \\
\hline RM22 & $\begin{array}{l}\text { Utilizar roupas condizentes com o cargo ocupado na organização, onde pessoas com mais } \\
\text { autoridade devem ter vestimentas mais formais, como gravatas. }\end{array}$ & 1 \\
\hline RM23 & Ter conhecimento adequado sobre as atividades das iniciativas de MPS. & 1 \\
\hline RM24 & Dedicar esforço para o sucesso da implementação de iniciativas de MPS. & 1 \\
\hline
\end{tabular}


A Tabela 5 apresenta as RMs relacionadas ao grupo fator social, ou seja, aquelas que alteram as estruturas sociais que compõem um grupo ou uma sociedade. Em outras palavras, o fator social ocorre quando as estruturas da sociedade sofrem uma transformação motivada pela ocorrência de fenômenos socioculturais e operam mudanças nos comportamentos, nas atitudes e nos sistemas de valores [Lacerda, 2011].

Tabela 5. RMs do fator social extraídas das publicações

\begin{tabular}{|c|c|c|}
\hline RMs & Descrição & Citações \\
\hline \multicolumn{3}{|c|}{ Clientes: } \\
\hline RM25 & $\begin{array}{l}\text { Responder constantemente às necessidades dos clientes e estabelecer um relacionamento de } \\
\text { confiança com eles. }\end{array}$ & 4 \\
\hline RM26 & Expor suas ideias e propostas ao cliente, ao invés de aceitar tudo que ele propõe. & 1 \\
\hline RM27 & Alinhar os interesses do cliente com os dos funcionários. & 1 \\
\hline \multicolumn{3}{|c|}{ Participacão: } \\
\hline RM28 & $\begin{array}{l}\text { Realizar reuniões periódicas dando ênfase aos benefícios que a organização tem adquirido com } \\
\text { a implementação da iniciativa de MPS e caracterizar a situação da organização no futuro. }\end{array}$ & 4 \\
\hline RM29 & $\begin{array}{l}\text { Criar possibilidades de todos participarem da tomada de decisões de forma a se ter um ambiente } \\
\text { no qual os profissionais sejam capazes de expressar suas opiniões. Um exemplo é garantir que } \\
\text { os funcionários participem na escolha das ferramentas ao invés de ser uma imposição quanto ao } \\
\text { seu uso e compra. }\end{array}$ & 3 \\
\hline RM30 & $\begin{array}{l}\text { Promover a satisfação e o envolvimento do funcionário através de um planejamento cuidadoso, } \\
\text { de um ambiente disciplinado, exigente e desafiante, com oportunidades de crescimento e } \\
\text { atribuições coerentes. Além disso, levar em consideração o bem-estar dos funcionários, tendo a } \\
\text { preocupação com a carga de trabalho pesada e más condições de trabalho. }\end{array}$ & 3 \\
\hline RM31 & $\begin{array}{l}\text { Realizar fóruns de discussão onde os funcionários possam expressar livremente suas idéias e } \\
\text { propostas para a implementação da iniciativa de MPS. Em alguns casos, deve ter mais } \\
\text { autonomia para fazer sugestões para o novo processo. Todas as idéias, mesmo as mais adversas } \\
\text { devem ser ouvidas, analisadas e discutidas com os que as propuseram. }\end{array}$ & 2 \\
\hline RM32 & Exigir a presença de todos os funcionários nas reuniões. & 2 \\
\hline RM33 & $\begin{array}{l}\text { Realizar reuniões fora do trabalho, cuidadosamente planejadas, para descontrair e estimular a } \\
\text { união da equipe. }\end{array}$ & 1 \\
\hline RM34 & Realizar seminários para comunicar as práticas do programa de MPS para os funcionários. & 1 \\
\hline RM35 & Incentivar a participação de voluntários na implementação da iniciativa de MPS. & 1 \\
\hline \multicolumn{3}{|c|}{ Apoio: } \\
\hline RM36 & $\begin{array}{l}\text { Mostrar claramente o apoio na implementação da iniciativa de MPS e não ser contraditória nas } \\
\text { decisões e ações tomadas. }\end{array}$ & 2 \\
\hline RM37 & Ter responsabilidades no apoio à iniciativa de MPS. & 1 \\
\hline RM38 & Contar com apoio de dentro e fora da organização. & 1 \\
\hline RM39 & $\begin{array}{l}\text { Convencer os funcionários da organização sobre a real necessidade de implementar uma } \\
\text { iniciativa de MPS. Isto pode ser feito através de uma reunião, apresentando análises estatísticas } \\
\text { sobre a situação da empresa e das concorrentes, possíveis crises e as melhorias que irão ocorrer } \\
\text { com a implementação da iniciativa de MPS. }\end{array}$ & 1 \\
\hline \multicolumn{3}{|c|}{ Comprometimento: } \\
\hline RM40 & $\begin{array}{l}\text { Ter consciência que o compromisso com programas de melhorias é a longo prazo. O } \\
\text { compromisso pode ser estabelecido através da cooperação interorganizacional, onde os } \\
\text { participantes podem ser envolvidos em uma competição amigável sobre o andamento da } \\
\text { iniciativa de MPS. Ou ainda, através de um bônus e novas oportunidades de carreira. }\end{array}$ & 8 \\
\hline RM41 & $\begin{array}{l}\text { Conquistar a confiança, a contribuição e o compromisso dos funcionários, além de promover o } \\
\text { diálogo. Isso pode ser feito através da participação dos mesmos nas decisões sobre MPS. } \\
\text { Consultar os funcionários quando decisões mais importantes forem tomadas. Argumentar que o } \\
\text { sucesso da iniciativa depende da participação de todos e envolvê-los na implementação da } \\
\text { iniciativa de MPS. }\end{array}$ & 4 \\
\hline RM42 & $\begin{array}{l}\text { Ter a capacidade de se adaptar dinamicamente a uma rápida mudança, até mesmo no mais } \\
\text { caótico ambiente. Adotar táticas apropriadas para o gerenciamento das mudanças ocorridas com } \\
\text { a iniciativa de MPS. Estar preparados para as mudanças, particularmente nas áreas de } \\
\text { planejamento, controle, procedimentos (principalmente para as definições de processos formais) } \\
\text { e cultura (adotar um conjunto de estratégias para gerir as mudanças ocasionadas pela } \\
\text { implementação de MPS). }\end{array}$ & 3 \\
\hline RM43 & $\begin{array}{l}\text { Estar envolvidos, os gerentes de projetos ou a pessoa responsável pela iniciativa de MPS, em } \\
\text { todas as suas fases de implementação. }\end{array}$ & 1 \\
\hline RM44 & $\begin{array}{l}\text { Assegurar que os funcionários estejam motivados a participarem da implementação da iniciativa } \\
\text { de MPS. }\end{array}$ & 1 \\
\hline
\end{tabular}




\begin{tabular}{|l|l|c|}
\hline RM45 & $\begin{array}{l}\text { Realizar uma avaliação do desempenho atual antes que as mudanças organizacionais comecem } \\
\text { com a implementação da iniciativa de MPS. }\end{array}$ & 1 \\
\hline RM46 & $\begin{array}{l}\text { Medir o progresso da mudança organizacional, realizando avaliações periódicas, o desempenho } \\
\text { dos processos e a satisfação das partes interessadas. }\end{array}$ & 1 \\
\hline RM47 & Verificar se os funcionários são resistentes à mudanças. & 1 \\
\hline RM48 & Estruturar as mudanças antes de serem anunciadas. & 1 \\
\hline \multicolumn{2}{|c|}{ Ambiente: } & 2 \\
\hline RM49 & $\begin{array}{l}\text { Propiciar um ambiente simples, aconchegante, organizado, descontraído, relaxante, acolhedor, } \\
\text { arrumado, limpo e com uma decoração elegante. }\end{array}$ & 1 \\
\hline RM50 & Definir um espaço próprio para os funcionários, que deve ser caracterizado com itens pessoais. & 1 \\
\hline RM51 & Propiciar um ambiente que tenha previsibilidade, confiabilidade e uniformidade (constância). & 1 \\
\hline RM52 & $\begin{array}{l}\text { Operar em um ambiente que tenha uma unidade integrada com um conjunto comum de normas, } \\
\text { crenças, ideias e valores. }\end{array}$ & 1 \\
\hline RM53 & Balancear a disciplina e a criatividade na forma de trabalho dos funcionários. & 1 \\
\hline
\end{tabular}

O fator organizacional está relacionado a qualquer ação ocorrida na organização, decorrente de fatores internos e/ou externos, que traz algum impacto nos resultados e/ou nas relações entre as pessoas no trabalho [Montana e Charnov, 1998]. A Tabela 6 apresenta as RMs distribuídas no grupo associadas a este fator.

\section{Tabela 6. RMs do fator organizacional extraídas das publicações}

\begin{tabular}{|c|c|c|}
\hline RMs & Descrição & Citações \\
\hline \multicolumn{3}{|c|}{ Políticas Organizacionais: } \\
\hline RM54 & Estabelecer um canal de comunicação eficaz e formal em toda a organização. & 6 \\
\hline RM55 & $\begin{array}{l}\text { Estabelecer políticas organizacionais e administrativas para controle e acompanhamento das } \\
\text { atividades de MPS. Gerenciar os prazos e os resultados estabelecidos para a iniciativa de MPS. } \\
\text { Os resultados obtidos (qualitativos e quantitativos) devem ser publicados de modo que todos os } \\
\text { funcionários tenham acesso. }\end{array}$ & 5 \\
\hline RM56 & $\begin{array}{l}\text { Criar contextos organizacionais que apoiam e favoreçam a aprendizagem organizacional e } \\
\text { integrá-los no dia-a-dia do trabalho da organização. }\end{array}$ & 2 \\
\hline RM57 & $\begin{array}{l}\text { Conhecer as políticas organizacionais da empresa e torná-las favoráveis à implementação da } \\
\text { estratégia de MPS. }\end{array}$ & 2 \\
\hline RM58 & Revisar periodicamente as atividades da iniciativa de MPS. & 2 \\
\hline RM59 & $\begin{array}{l}\text { Ter estabilidade e controle através de tarefas claras e bem definidas com aplicação de regras } \\
\text { rigorosas. }\end{array}$ & 2 \\
\hline RM60 & $\begin{array}{l}\text { Adotar práticas de MPS ágeis para assegurar o ajuste organizacional com as novas } \\
\text { circunstâncias que cercam a iniciativa de MPS. }\end{array}$ & 2 \\
\hline RM61 & Gerenciar o crescimento organizacional na capacidade dos funcionários. & 1 \\
\hline RM62 & Estabelecer crenças ou princípios que irão apoiar a implementação da iniciativa de MPS. & 1 \\
\hline RM63 & Definir um modelo de negócios e seus procedimentos formais. & 1 \\
\hline RM64 & $\begin{array}{l}\text { Adotar táticas apropriadas para o estabelecimento de uma ligação entre o processo e os } \\
\text { objetivos de negócio da organização. }\end{array}$ & 1 \\
\hline RM65 & $\begin{array}{l}\text { Eliminar os problemas que impedem os funcionários de serem capazes de executar suas } \\
\text { responsabilidades de forma eficaz. }\end{array}$ & 1 \\
\hline RM66 & $\begin{array}{l}\text { Demitir pessoas cujo comportamento apresenta riscos para o sucesso da implementação do } \\
\text { programa. }\end{array}$ & 1 \\
\hline \multicolumn{3}{|c|}{ Metas/Objetivos: } \\
\hline RM67 & $\begin{array}{l}\text { Definir, coletivamente, as metas, os objetivos, as estratégias e os recursos necessários para } \\
\text { implementação da iniciativa de MPS, tanto a curto quanto á longo prazo, e alinhá-los com os } \\
\text { objetivos e as estratégias da organização, deixando-os explícitos a todos os envolvidos na } \\
\text { organização, assegurando que estes compreendam a razão da iniciativa de MPS. Definir um } \\
\text { calendário de atividades da iniciativa de MPS. }\end{array}$ & 9 \\
\hline RM68 & $\begin{array}{l}\text { Medir e comunicar o andamento da implementação da iniciativa de MPS. Realizar as medições } \\
\text { e as avaliaçóes de desempenho de forma confiável e com métodos válidos. }\end{array}$ & 7 \\
\hline RM69 & $\begin{array}{l}\text { Definir e articular a visão da nova organização, moldar sua cultura, determinar os parâmetros de } \\
\text { sucesso e informar como ela irá interagir com os principais interessados. }\end{array}$ & 1 \\
\hline RM70 & $\begin{array}{l}\text { Colocar em evidência, em uma placa, quadro ou lousa, todos os objetivos da iniciativa de MPS, } \\
\text { como artefatos, metas pré-estabelecidas, processos, etc. Cada conquista deve ser destacada, bem } \\
\text { como as pessoas que ajudaram na sua obtenção. }\end{array}$ & 1 \\
\hline \multicolumn{3}{|c|}{ Cultura Organizacional: } \\
\hline RM71 & Alinhar a cultura organizacional com o gerenciamento de projetos de software. & 3 \\
\hline RM72 & $\begin{array}{l}\text { Divulgar a cultura organizacional através de suas crenças e costumes organizacionais para todos } \\
\text { os membros da organização. }\end{array}$ & 2 \\
\hline
\end{tabular}




\begin{tabular}{|c|c|c|}
\hline RM73 & $\begin{array}{l}\text { Utilizar os insights da cultura organizacional para melhor implementarem a iniciativa de MPS, } \\
\text { isto é, assegurar que as práticas de MPS estejam de acordo com a cultura organizacional. }\end{array}$ & 2 \\
\hline RM74 & $\begin{array}{l}\text { Concentrar esforços para criar uma cultura organizacional própria em que os procedimentos } \\
\text { técnicos e organizacionais possam prosperar. }\end{array}$ & 1 \\
\hline RM75 & $\begin{array}{l}\text { Realizar uma análise do impacto da cultura organizacional sobre os interesses nas iniciativas de } \\
\text { MPS e vice-versa. }\end{array}$ & 1 \\
\hline RM76 & $\begin{array}{l}\text { Entender as diferenças culturais subjacentes às práticas de desenvolvimento de software e } \\
\text { adaptá-las às iniciativas de MPS, principalmente quando se tratar de uma organização } \\
\text { multinacional. }\end{array}$ & 1 \\
\hline RM77 & $\begin{array}{l}\text { Estabelecer uma cultura que internalize os valores profissionais da organização e garanta o } \\
\text { cumprimento das decisões tomadas em relação à qualidade dos processos. }\end{array}$ & 1 \\
\hline \multicolumn{3}{|c|}{ Investimentos/Recursos: } \\
\hline RM78 & Investir em tecnologias, cursos e ferramentas que suportam a execução de iniciativas de MPS. & 10 \\
\hline RM79 & Alocar e priorizar recursos para investir especificamente na iniciativa de MPS. & 3 \\
\hline RM80 & $\begin{array}{l}\text { Dar recompensas e benefícios, como dias de folga, reconhecimento ou itens materiais, para os } \\
\text { funcionários mais envolvidos e que contribuíram para a iniciativa. }\end{array}$ & 2 \\
\hline RM81 & Estabelecer novas estruturas necessárias para garantir a gestão e controle. & 1 \\
\hline RM82 & Alocar recursos para evitar desânimo ou decepção nos funcionários. & 1 \\
\hline RM83 & $\begin{array}{l}\text { Utilizar modelos que verificam se uma tecnologia é bem aceita e tem facilidade de uso e } \\
\text { modelos que explicam o comportamento humano. }\end{array}$ & 1 \\
\hline RM84 & Investir em gestão de conhecimento. & 1 \\
\hline RM85 & Ter uma infraestrutura forte e eficaz. & 1 \\
\hline RM86 & $\begin{array}{l}\text { Contratar consultores externos e avaliadores, caso o conhecimento necessário para implementar } \\
\text { uma iniciativa de MPS não exista dentro da organização. }\end{array}$ & 1 \\
\hline \multicolumn{3}{|c|}{ Planos/Planejamentos: } \\
\hline RM87 & $\begin{array}{l}\text { Ter um plano estratégico para resolver problemas que possam ocorrer com a iniciativa de MPS. } \\
\text { Neste plano devem constar ações de intervenção, de assistência e garantia de melhores } \\
\text { condições de trabalho. Além disso, analisar fatores situacionais em termos dos riscos } \\
\text { enfrentados por uma equipe de MPS. }\end{array}$ & 5 \\
\hline RM88 & $\begin{array}{l}\text { Ter um plano de estabilidade no emprego (plano de carreira) para os funcionários através da } \\
\text { definição de regras e divulgar entre eles. }\end{array}$ & 3 \\
\hline RM89 & $\begin{array}{l}\text { Adequar as práticas da força de trabalho para desenvolver habilidades e competências } \\
\text { específicas que a organização necessita. }\end{array}$ & 3 \\
\hline RM90 & Criar planos de ação para a implementação da iniciativa de MPS. & 2 \\
\hline RM91 & $\begin{array}{l}\text { Planejar um conjunto de cursos sobre temas relacionados com iniciativas de MPS e no uso de } \\
\text { ferramentas estatísticas. }\end{array}$ & 2 \\
\hline RM92 & $\begin{array}{l}\text { Incluir um plano de gerenciamento, com disponibilidade de recursos financeiros para cursos de } \\
\text { qualificação, incentivos financeiros significativos e um maior investimento em funcionários que } \\
\text { demonstram mais competência. }\end{array}$ & 2 \\
\hline RM93 & $\begin{array}{l}\text { Elaborar um plano de incentivo para induzir mudanças no comportamento e desempenho dos } \\
\text { funcionários. }\end{array}$ & 2 \\
\hline RM94 & $\begin{array}{l}\text { Definir um organograma, definindo claramente uma hierarquia entre os funcionários e } \\
\text { informá-los. }\end{array}$ & 1 \\
\hline RM95 & Ter um plano de ação para evitar ou corrigir erros. & 1 \\
\hline RM96 & $\begin{array}{l}\text { Ter foco na melhoria de processos para ajudar a esclarecer as expectativas dos colaboradores e } \\
\text { contribuir para a satisfação no trabalho. }\end{array}$ & 1 \\
\hline RM97 & Reduzir a rotatividade dos funcionários. & 1 \\
\hline RM98 & Adotar um plano para garantir a divulgação do processo. & 1 \\
\hline RM99 & Ter controle sobre os funcionários que estão sob sua direção. & 1 \\
\hline RM100 & Ter as descrições dos processos de melhoria bem definidos, o que deve ser feito e como fazê-lo. & 1 \\
\hline
\end{tabular}

\section{Análise das Recomendações de Melhoria}

A análise das RMs foi feita a partir dos dados obtidos na RS e que foram descritos na Seção 3. Esta análise visa responder às questões secundárias de pesquisa apresentadas na Seção 2, além de análises baseadas nos grupos (fatores) e suas categorias.

\section{- RMs Identificadas que Promoveram o Sucesso da Iniciativa de MPS}

Nenhuma publicação citou se as RMs sugeridas promoveram ou não o sucesso na adoção/implementação da iniciativa de MPS. 


\section{- Evidência de que as RMs foram Avaliadas}

Cinco publicações (45\%) apresentaram evidências de que as RMs foram avaliadas, sejam por pesquisa de opinião [Dyba, 2005], estudos de casos [Mathiassen et al., 2005 ; Iversen e Ngwenyama, 2006] ou revisões da literatura [Ferreira e Wazlawick, 2011 ; Muller et al., 2010].

\section{- RMs por Número de Citações}

Das 100 RMs identificadas, dez se destacaram por terem sido citadas por mais de $45 \%$ dos 11 trabalhos identificados na Tabela 3. Dentre elas, 6 estão relacionadas com o FO, 3 fazem parte do grupo FH e 1 está associada ao FS. Este resultado pode sugerir que estas são as RMs mais relevantes de acordo com a perspectiva investigada nesta pesquisa. As dez RMs mais citadas estão listadas na Tabela 7.

Tabela 7. Lista das 10 RMs mais citadas

\begin{tabular}{|c|l|c|c|}
\hline ID & \multicolumn{1}{|c|}{ A Recomendação de Melhoria Está Relacionada a... } & Grupo & \% de Citações \\
\hline RM1 & Estabelecimento de uma equipe de garantia de qualidade & FH & $100 \%(11 / 11)$ \\
\hline RM78 & Investimentos em ferramentas e cursos de apoio a iniciativa de MPS & FO & $91 \%(10 / 11)$ \\
\hline RM67 & $\begin{array}{l}\text { Forma de como deve ser feita a definição dos objetivos e metas da iniciativa } \\
\text { de MPS }\end{array}$ & FO & $82 \%(9 / 11)$ \\
\hline RM2 & Papéis na implementação da iniciativa de MPS & FH & $73 \%(8 / 11)$ \\
\hline RM40 & Compromisso com a iniciativa de MPS & FS & $73 \%(8 / 11)$ \\
\hline RM68 & Medições e avaliações de desempenho & FO & $64 \%(7 / 11)$ \\
\hline RM54 & Estabelecimento de um canal de comunicação & FO & $55 \%(6 / 11)$ \\
\hline RM55 & $\begin{array}{l}\text { Políticas organizacionais e administrativas para controle e acompanhamento } \\
\text { das atividades de MPS }\end{array}$ & FO & $46 \%(5 / 11)$ \\
\hline RM87 & $\begin{array}{l}\text { Plano estratégico para resolver problemas que possam ocorrer com a } \\
\text { iniciativa de MPS }\end{array}$ & FO & $46 \%(5 / 11)$ \\
\hline RM18 & Definição dos líderes responsáveis pela implementação da iniciativa de MPS & FH & $46 \%(5 / 11)$ \\
\hline
\end{tabular}

A Figura 2 mostra a distribuição das 100 RMs pelo número de citações, separadas por faixas: com 1 citação, com 2 a 4, com 5 a 7 e com 8 a 11 citações. $O$ valor exato de citações para cada RM está apresentado nas Tabelas 4, 5 e 6 . Apesar da maioria das RMs ter apenas 1 citação, não se pode concluir que estas RMs não sejam relevantes. É possível que, em determinadas organizações, a ausência dessas RMs seja capaz de exercer influência direta no sucesso da iniciativa de melhoria.

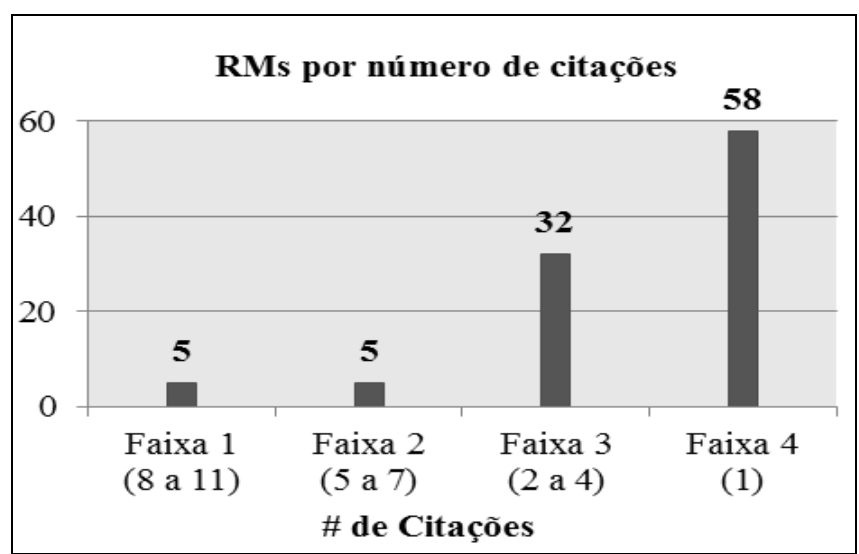

Figura 2. Distribuição das 100 RMs pelo número de citações 


\section{- RMs Distribuídas por Fator Humano, Social e Organizacional}

É possível observar que $24 \%$ das 100 RMs estão associadas ao fator humano, 29\%, ao fator social e $47 \%$, ao fator organizacional, conforme a Figura 3. Isso sugere que ao pensar em melhorias relacionadas a aspectos culturais de uma organização, alternativas para problemas administrativos e gerenciais são os mais discutidos pela literatura técnica. Este resultado reforça que a compreensão de questões organizacionais é fundamental para alcançar o alinhamento entre as atividades da iniciativa de MPS e os objetivos da organização, conforme já discutido no trabalho de [Dyba, 2002].

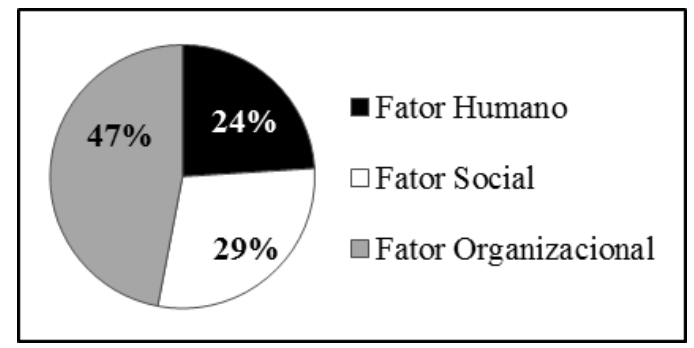

Figura 3. Distribuição das 100 RMs por fatores

\section{- RMs Distribuídas por Categorias}

Esta análise foi realizada a partir das categorias nas quais as RMs foram subdivididas dentre seus grupos (FH, FS e FO). Os resultados indicam que as categorias "Planos/Planejamentos" (Fator Organizacional), "Políticas Organizacionais" (Fator Organizacional) e "Equipes de Trabalho" (Fator Humano) são as que mais apresentam RMs, conforme Figura 4. Essas três categorias, juntas, concentram 37 RMs (37\%) o que reforça a importância da organização de apresentar um planejamento adequado antes da implementação da iniciativa de melhoria [EspinosaCuriel et al., 2011], de criar contextos organizacionais que favoreçam essa iniciativa [Niazi, 2012] e de definir as competências e as responsabilidades dos membros das equipes de trabalho que serão envolvidas durante o processo de implementação da iniciativa de MPS [Montoni e Rocha, 2011].

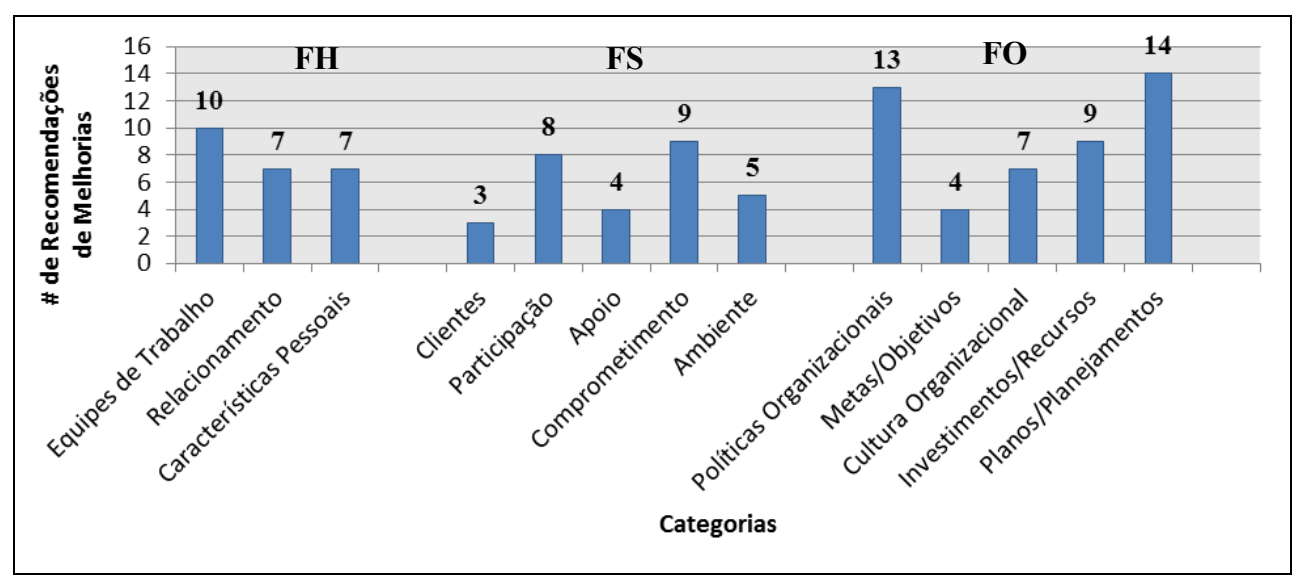

Figura 4. Distribuição das 100 RMs por categorias 


\section{Conclusões e Trabalhos Futuros}

Diversas empresas desenvolvedoras de software estão preocupadas em melhorar a qualidade de seus produtos, entregando-os de acordo com o orçamento e o prazo estabelecido. Uma das possíveis soluções é a adoção de uma iniciativa de MPS. Porém, somente algumas organizações conseguem implementar com sucesso uma iniciativa de MPS, devido a problemas organizacionais. Uma alternativa para evitar esses problemas é verificar em trabalhos publicados as recomendações de melhoria, ou relatos de experiências, que podem fornecer possíveis orientações relacionadas com a implementação dessas iniciativas.

Este trabalho realizou um estudo baseado em uma RSL com o objetivo de identificar RMs para apoiar as organizações de software a obterem sucesso na implementação de uma iniciativa de MPS. Com a execução da RSL, foi possível identificar 100 RMs que foram organizadas em grupos relacionadas a 3 fatores humano, social e organizacional - que influenciam na implementação de uma iniciativa de MPS e podem ajudar as organizações a reduzirem problemas e riscos, a conduzi-las a uma gestão mais eficiente e direcioná-las no alcance de seus objetivos e metas. Além disso, estas RMs podem auxiliar os diretores e gerentes na definição do planejamento estratégico, no gerenciamento dos recursos, nas mudanças e no melhoramento do desempenho da organização durante a implementação da iniciativa de MPS.

A organização deve garantir que todos os funcionários, de todos os níveis (alta direção, diretores, líderes de projeto e desenvolvedores), se comprometam a seguir as RMs e que estejam envolvidos na sua execução.

Em relação a perspectivas futuras, entende-se que esta pesquisa apresenta-se como um instrumento que pode contribuir na implementação de uma iniciativa de MPS. Porém, é necessário realizar estudos de casos, com organizações de software e equipes de desenvolvimento, colocando em prática as RMs apresentadas neste trabalho, para comprovar sua eficiência no sucesso da iniciativa de MPS. Além disso, poderia ser verificado, em estudos e trabalhos relacionados, como as RMs podem ser implantadas na organização de software. Diante disso, uma outra sugestão é separar as RMs por grupos que indicam o tipo de profissional da organização responsável pela sua implantação.

Não obstante, durante a leitura das publicações identificadas (Tabela 3), foi possível perceber, como sugestões de trabalhos futuros, que novas pesquisas deveriam ser realizadas sobre a gestão de iniciativas de MPS em contextos organizacionais, pois estas são pelo menos tão importantes para a MPS quanto à tecnologia.

\section{Agradecimentos}

À Fundação de Amparo à Pesquisa do Estado do Amazonas (FAPEAM) pelo apoio financeiro.

\section{Referências}

Barcellos, M.; Rocha, A. e Falbo, R. (2010). Uma Estratégia para Medição de Software e Avaliação de Bases de Medidas para Controle Estatístico de Processos de Software em Organizações de Alta Maturidade. IX Simpósio Brasileiro de Qualidade de Software, p. 409-423. 
Barreto, A. e Rocha, A. (2012). Definição e Gerência de Objetivos de Software Alinhados ao Planejamento Estratégico. XI Simpósio Brasileiro de Qualidade de Software, p. 399-413.

Biolchini, J. et al. (2005). Systematic Review in Software Engineering. Relatório Técnico RT-ES 679/05, Programa de Engenharia de Sistemas e Computação, Universidade Federal do Rio de Janeiro, Rio de Janeiro.

Cameron, K. e Quinn, R. (2006). Diagnosing and Changing Organizational Culture: Based on The Competing Values Framework. Revised Edition. The Jossey-Bass Business \& Management Series.

Dyba, T. (2002). Enabling Software Process Improvement: An Investigation of the Importance of Organizational Issues. Empirical Software Engineering, v. 7, p. 387390.

Dyba, T. (2005). An Empirical Investigation of the Key Factors for Success in Software Process Improvement. IEEE Transactions on Software Engineering, v. 31, n. 5, p. 410-424.

Elsevier. (2013) What does Scopus cover?. Acessado em Março de 2013. Disponível em $<$ http://www.info.sciverse. com/scopus/scopus-in-detail/facts $>$.

Espinosa-Curiel, I.; Rodríguez-Jacobo, J. e Fernández-Zepeda, J. (2011). A Framework for Evaluation and Control of the Factors that Influence the Software Process Improvement in Small Organizations. Journal of Software Maintenance and Evolution Research and Practice, v. 25.

Ferreira, M.; Wazlawick, R. (2011). Software Process Improvement: A Organizational Change that Need to be Managed and Motivated. World Academy of Science, Engineering and Technology, v. 50, p. 296-304.

ISO/IEC. (2003). ISO/IEC 15504: Information Technology - Process Assessment. International Standard.

ISO/IEC. (2008). ISO/IEC 12207: System and Software Engineering - Software Life Cycle Processes. Genene: ISO.

Iversen, J. e Ngwenyama, O. (2006). Problems in Measuring Effectiveness in Software Process Improvement: A Longitudinal Study of Organizational Change at Danske Data. International Journal of Information Management, v. 26, p. 30-43.

Kitchenham, B. (2004). Procedures for Performing Systematic Reviews, Technical Report, Departament of Computer Science Keele University, Keele.

Lacerda, F. (2011). Psicologia cultural e mudança social. Psicologia e Sociedade, v. 23, n. 1, p. 197-200.

Mathiassen, L.; Ngwenyama, O. e Aaen, I. (2005). Managing Change in Software Process Improvement. IEEE Software, v. 22, n. 6, p. 84-91.

Montana, P. e Charnov, B. (1998). Administração. São Paulo: Saraiva.

Montoni, M. e Rocha, A. (2011). Uma Investigação sobre os Fatores Críticos de Sucesso em Iniciativas de Melhoria de Processos de Software. X Simpósio Brasileiro de Qualidade de Software, p. 151-165.

Muller, S.; Kraemmergaard, P. e Mathiassen, L. (2009). Managing Cultural Variation in Software Process Improvement: A Comparison of Methods for Subculture Assessment. IEEE Transactions on Engineering Management, v. 56, n. 4, p. 584-599. 
Muller, S.; Mathiassen, L. e Balshoj, H. (2010). Software Process Improvement as Organizational Change: A Metaphorical Analysis of the Literature Journal of Systems and Software, v. 83, n. 11, p. 2128-2146.

Niazi, M. (2012). An Exploratory Study of Software Process Improvement Implementation Risks. Journal of Software Maintenance and Evolution: Research and Practice, v. 24, p. 877-894.

Passos, O.; Dias-Neto, A. e Barreto, R. (2012). Revisão Sistemática sobre Recomendações de Melhoria Baseadas na Cultura Organizacional para Iniciativas de Melhorias de Processo de Software. Relatório Técnico UFAM/GISE RT-004/2013, Programa de Pós-Graduação em Informática, Universidade Federal do Amazonas, Manaus.

Petersen, K. et al. (2008). Systematic Mapping Studies in Software Engineering. Evaluation and Assessment in Software Engineering (EASE), p. 1-10, Bari, Italy.

Ricardo, M. e Correa, A. (2011). MPS.BR Nível D - A Experiência em Implantar o Modelo na Área de Governo Municipal. Workshop Anual do MPS, p.94-103.

Rodrigues, J. e Kirne, T. (2010). Benefícios, Fatores de Sucesso e Dificuldades da Implantação do Modelo MPS.Br. IX Simpósio Brasileiro de Qualidade de Software, p. 41-55.

Santos, G. (2008). Ambientes de Engenharia de Software Orientados à Corporação. Tese de Doutorado, COPPE/UFRJ, Rio de Janeiro, RJ, Brasil.

Santos, G. (2011). Influência e Impacto do Programa MPS.Br na Pesquisa Relacionada à Qualidade de Software no Brasil. X Simpósio Brasileiro de Qualidade de Software, p. 73-87.

Schein, E. (2009). Cultura Organizacional e Liderança. 3. ed. São Paulo: Atlas.

SEI. (2006). CMMI para Desenvolvimento (CMMI-DEV), versão 1.2, CMU/SEI-2006TR-008. Software Engineering Institute.

Shih, C-C. e Huang, S-J. (2010). Exploring the Relationship Between Organizational Culture and Software Process Improvement Deployment. Information \& Management, v. 47, p. 271-281.

SOFTEX - Associação para Promoção da Excelência do Software Brasileiro. (2009). MPS.BR - Melhoria de Processo do Software Brasileiro - Guia Geral, Guia de Implementação e Guia de Avaliação.

Souza, C. et al. (2009). Avaliação Conjunta CMMI Nível 3 e MPS Nível C: Lições Aprendidas e Recomendações. Workshop Anual do MPS, p. 52-61.

Souza, W. et al. (2010). MPS.BR Nível A: Experiência da Stefanini. Workshop Anual do MPS, p. 128-137.

Zoucas, A.; Thiry, M. e Cunha, C. (2012). Compreendendo a Influência da Liderança nas Iniciativas de Melhoria de Processo de Desenvolvimento de Software. XI Simpósio Brasileiro de Qualidade de Software, p. 274-288. 